\title{
Research and Design On Dynamic Weighing System with Piezoelectric Polymer Sensor

\author{
Hai Guan ${ }^{2, \mathrm{~d}}$ and Tieyi Zhang ${ }^{1, \mathrm{e}}$
} \\ Jun Wei ${ }^{1, a}$, Jinquan Xie ${ }^{1, b}$, Ligeng Qin ${ }^{2, c}$,
}

${ }^{1}$ School of .Mechanical Engineering, Guangxi University, Nanning 530004, China

${ }^{2}$ Guangxi Transportation Research Institute, Nanning 530007, China

a1514236866@qq.com , b754205880@qq.com, c $46557560 @ q q . c o m$,

d691630423@qq.com , e zhangty-1@163.com

Keywords: piezoelectric polymer sensor, dynamic weighing, area integral

Abstract: A new dynamic weighing system with piezoelectric polymer sensor which includes hardware, software and algorithm is researched and designed .Then the preliminary trial, conducted in the designing system, and the analysis about experimental results, verify the feasibility and effectiveness of the system. The researched results help to obtain the normal and efficient operation of the dynamic weighing system for a long time.

\section{Introduction}

With the rapid development of our national economy and transportation business, the number of heavy loaded trucks on the road increases rapidly. At same time the overload vehicles arises which has become a intractable problem. To protect the road from overload damage, dynamic weighting is widely used in many conditions. Random load and impact load generated in vehicle movement lead to the complicity of vehicle movement, As a result, they bring also many problems to the dynamic weighing [1]. In order to design a dynamic weighing system a proper weigh sensor should be chosen carefully, which can reduce or eliminate the interference. At the same time, choosing the proper amplifier for amplifying the sampled signal and adopting reasonable algorithm for data processing will eventually control the measurement error within specified rang and increase weighing precision.

\section{The Dynamic Weighing System Design}

Dynamic weighing system hardware circuit mainly includes axle load sensor, charge amplifier and the PC, it is shown in Fig.1.

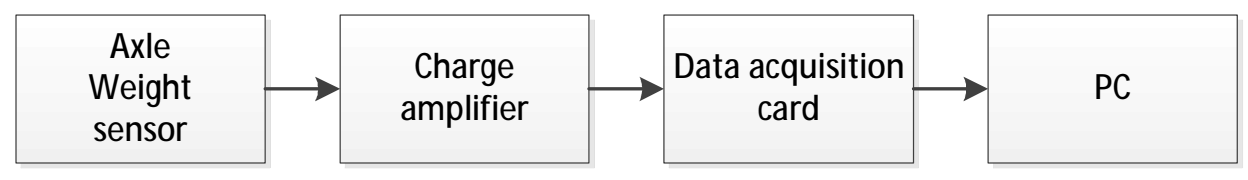

Fig.1 Diagram of hardware circuit

Firstly, the weight of the vehicle axle load signal will be changed into a weak charge Q by sensors. Then the charge amplifier will change $Q$ into voltage which is proportional to $Q$, and then voltage is converted to digital signal by the data acquisition card and input into an industrial computer which has a better anti-interference ability than normal personal computer. The weight of the vehicle axle calculation will be finished by the industrial computer. The industrial computer is shown in Fig. 2. 


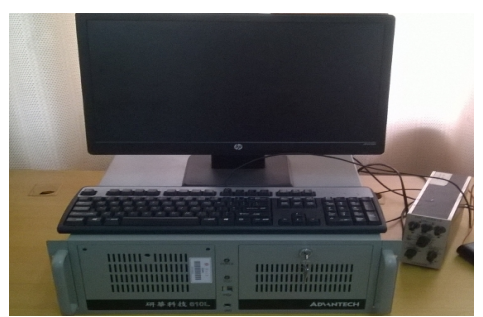

Fig.2 Industrial computer

The Selection of Weighing Sensor In current dynamic weighing system, there are the following kinds of Weighing Sensor such as bending plate sensor, the co-polymerization of compound type piezoelectric sensor, capacitive sensor, bridge type weighing platform, optical fiber sensor, the integrated intelligent digital weighing sensor etc. [2,3].

Co-polymerization of compound type piezoelectric sensor is chosen as weighing sensor in the system after comparison in above weighting sensors, it has the following advantages such as (1) quite a wide range of frequencies,(2) good dynamic characteristics;(3) high dielectric strength compared with piezoelectric ceramic materials which can exert high electric field strength;(4) has the high electrical impedance[4].

The above advantages of piezoelectric thin film shaft sensor meet the needs of conversion and delivery requirements of dynamic weighing system signal, It improves the dynamic precision of the dynamic weighing system and is benefit to reduce the weighing error.

Sensor Encapsulation and Laid In the dynamic weighing system, size of piezoelectric thin film for strip sensor shaft sensor used is $6 \mathrm{~mm} * 1 \mathrm{~mm} * 2000 \mathrm{~mm}$. A "sandwich" type of sensor encapsulation method is designed according to the characteristics of the piezoelectric polymer sensor,. Firstly, the piezoelectric polymer sensor is fixed on a piece of soft rubber mat with epoxy resin, and then a layer of soft rubber pad is covered on it. The sensor encapsulation is shown in Fig. 3.

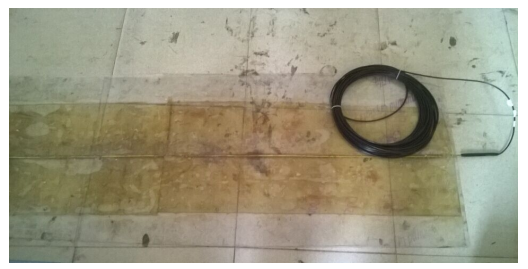

Fig.3 Sensor Encapsulation

Data Processing Algorithm At present, the basic algorithm of dynamic weighing system of piezoelectric thin film shaft are peak method and area of integral method. In this system, area integral method is applied as weighing system data processing algorithms. the process of vehicle tire moving and pressing on weighing sensor can be simplified as following. When the vehicle tire contacts with the sensor it produces a rapid linear ramp up of sensor's output voltage and soon reached the maximum value, which is proportional to the entire pressing load to the sensor. In the process when the wheel leaves the sensor, there will be a quick linear attenuation in the sensor's output. The sensor's output waveform is shown in Fig 4.

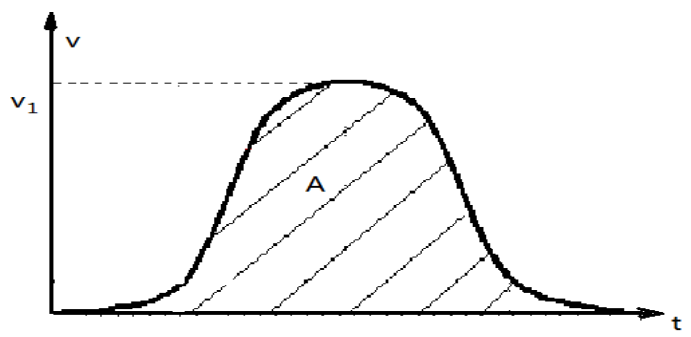

Fig. 4 The sensor's output waveform 
With the increase of vehicle speed, the time wheel passing through the weighing sensor will be shortened accordingly, and the waveform of sensor output signal become narrow, the speed of rising and falling of the waveform increases, the area between signal waveform and transverse axis decreases. But the product of the area and the corresponding speed remains as a fixed value. This value is proportional to vehicle weighing load. On this basis, area integral method to measurement and calculate axle load with dynamic weighing sensor are shown in Eq.(1).

$$
\mathrm{W}=\mathrm{S} * \mathrm{C}
$$

Where $\mathrm{S}$ is the products the area under the curve $\mathrm{A}$ and the vehicle speed $\mathrm{V}, \mathrm{C}$ is an adjusted factor related to the sensor characteristics. It can be determined by test which use a known weight $\mathrm{W}_{\mathrm{r}}$ vehicles passing through piezoelectric thin film . after the test, the factor $\mathrm{C}$ is determined as $\mathrm{C}=\mathrm{W} / \mathrm{S}$.

Dynamic Weighing System Software Design Vehicle dynamic weighing system software design mainly includes: main program design, data collection, data processing and display, etc. When designing the software, modular, customization, openness and network, the principle of practical economy, should be followed [5]. In this weighing system, Labview is used to program software, the real-time data is stored as binary engineering file format(LVM), and then deal with real-time data of binary engineering documents. The program flowchart is shown in Fig 5.

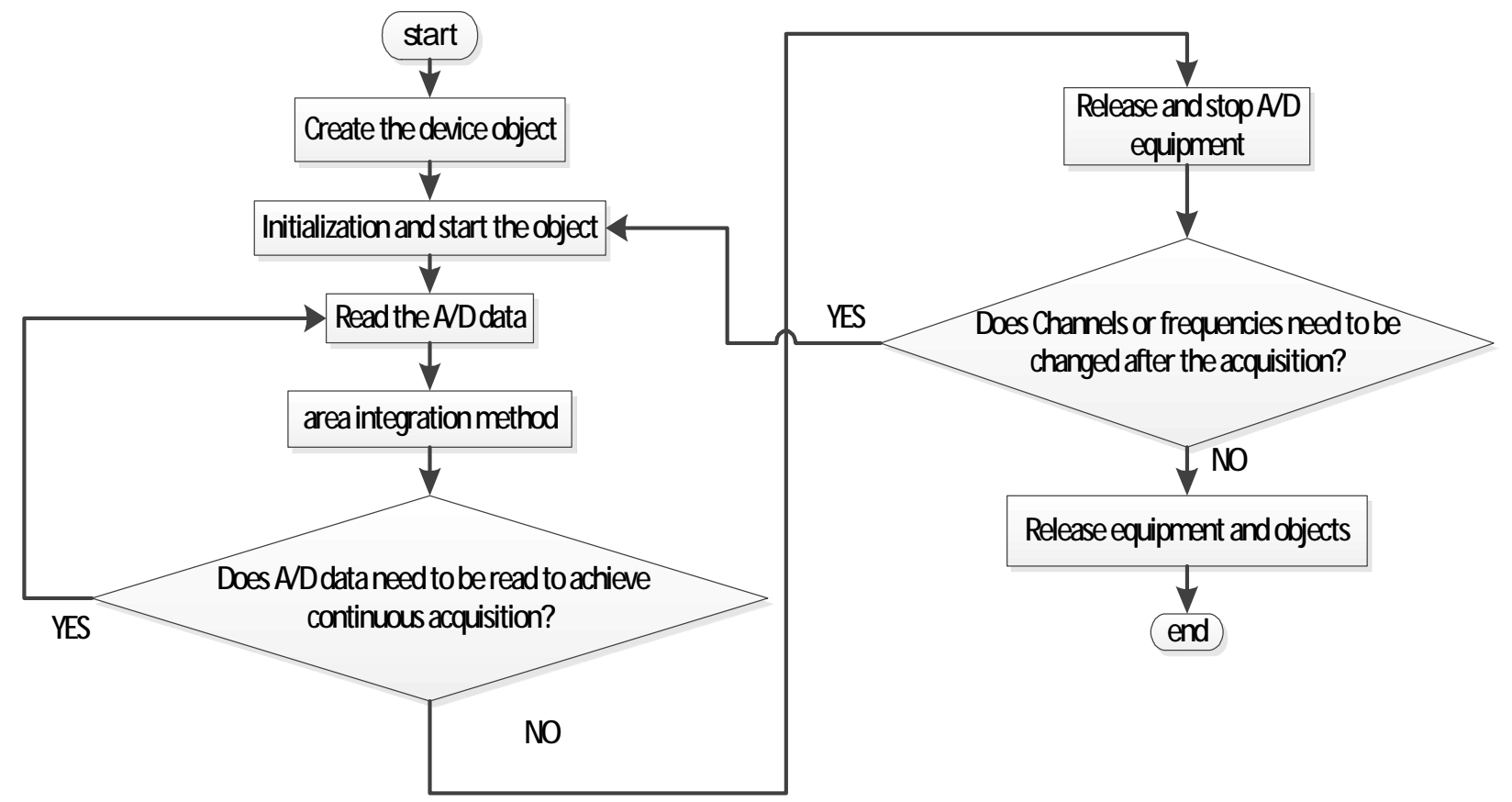

Fig. 5 The program flowchart

\section{Test and Results}

A good cement road is chosen on which the traffic is small on our campus to carried on the experiment after completion of the system design, First of all, piezoelectric thin film shaft sensor is firmly pasted on the pavement by using epoxy resin encapsulation to avoid the larger vibration and displacement when the experimental vehicle passes on the sensor. And then make sure the related instrument is in good connection. A storage battery driven bicycle is chosen as the test vehicle .Its static weighing of front and rear axle is measured with load weighing platforms. When the test vehicle pass though and press on sensors in different stable speed each time. The signal and result is recorded in the WIM system. Then change the weight of vehicle by carrying different number of person, repeat the test procedure and record the results. In the test, infrared velocemeter is used to measure the test speed in order to get the speed of the vehicle more accurately. After the test finished, part of the test analysis data is shown in the Table 1: 
Table 1: test data with area integral method

\begin{tabular}{|c|c|c|}
\hline Velocity $\mathrm{V}(\mathrm{km} / \mathrm{h})$ & The area of the pulse waveform in $\mathrm{S}$ & $\mathrm{V} * \mathrm{~S}$ \\
\hline 6 & 0.11000 & 0.660 \\
\hline 9 & 0.07700 & 0.693 \\
\hline 12 & 0.06000 & 0.720 \\
\hline 15 & 0.05067 & 0.750 \\
\hline 20 & 0.03920 & 0.784 \\
\hline 25 & 0.03168 & 0.792 \\
\hline 30 & 0.02660 & 0.798 \\
\hline 36 & 0.02206 & 0.794 \\
\hline 40 & 0.01945 & 0.778 \\
\hline 42 & 0.01850 & 0.777 \\
\hline
\end{tabular}

It is shown in the table that product of the speed of vehicle and pulse waveform in area is almost a constant value with only a small deviation which is within the error range. The conclusion that method of integrated area to calculate the vehicle axle load has higher precision which meet the practical requirement of dynamic weighing can be draw from the test .

\section{Conclusion}

The feasibility of the design of this dynamic weighing system and effectiveness is verified with experiment .The test results show the system has higher precision and measure error is in the allowable range. due to the experimental conditions is limited, such as the experimental vehicle is weight enough, speed is also not stable enough, These conditions can affect the performance of piezoelectric thin film shaft sensor and then affect the weighing accuracy of the system. In the future study, improving the test conditions, the system stability and weighing precision will be carried out.

\section{Acknowledgment}

The research is supported by Guangxi Key Project of Science and Technology (Granted No. 135500810) and supported by the opening project of Guangxi Colleges and Universities Key Laboratory of Modern Design and Advanced Manufacturing, The research is carried out with Guangxi Transportation Research Institute and Guangxi Nanning Jiesai Digital Technical Company.

\section{Reference}

[1] Li Xiaobao, Jane, Jane Water Peng Hui Xiang. Piezoelectric Quartz Weighing Sensor In The Application Of Highway Dynamic Weighing [J]. Journal Of Highway And Trucks: 2012. (2) : 9294.

[2] Wu Jisheng, Wang Dan, ETC. The Vehicle Dynamic Weighing System Based On ETC AxleLoad Data Processing Algorithm Research [J]. Highway Traffic Science And Technology, 2009 (10) : 190-194

[3] Zhang, Wang Qi Song-Lin Ma, Xin-Kai Li. A Novel Multi-Sensor Vehicle Classification System. Journal Of Tianjin University, 2008 (41) : 194-198.

[4] Shi Hang. Dynamic Weighing System Based On Piezoelectric Thin Film Shaft [D]. Jiangmen: Information Institute Of Wuyi University, 2006.

[5] Jia Haiqing Xinxing, High Snow Pool, Wang Lin. Dynamic Weighing System Data Acquisition And Analysis [J]. Highway Traffic Science And Technology, 2010 (6) : 138-14 